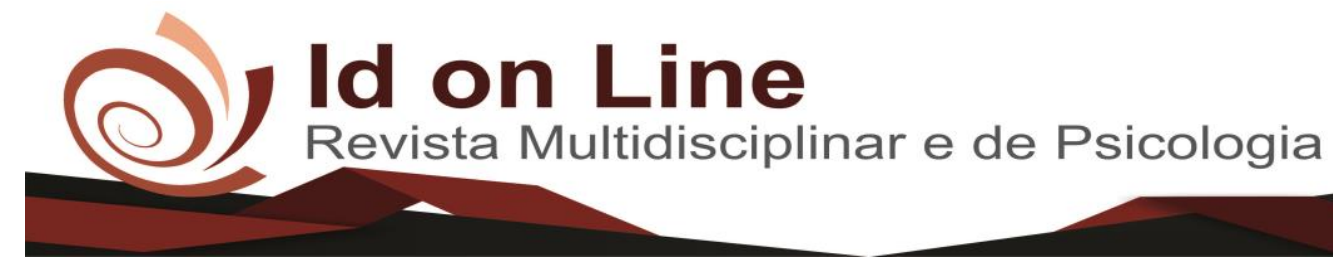

DOI: 10.14295/idonline.v15i56.3107

Artigo

\title{
A Discalculia e sua Influência na Aprendizagem da Química: Um Estudo de Caso em duas Escolas de Ensino Médio no Distrito Federal - DF
}

José Francisco de Sousa ${ }^{1}$

\begin{abstract}
Resumo. À partir do surgimento das neurociências descobriu-se como o funcionamento do cérebro influencia a educação, e consequentemente, a aprendizagem e com isso auxiliar professores e pesquisadores. Nesse sentido, o presente trabalho tem como objetivo analisar a relação entre os distúrbios de aprendizagem matemática e sua consequente influência no entendimento da Química e sua aprendizagem uma vez que esta área do conhecimento necessita para o seu entendimento de cálculos, sendo esta a hipótese de pesquisa. Trata-se de uma estudo descritivo, quantitativo, realizado com dados de 400 alunos estudantes do ensino médio de ambas as séries de duas escolas públicas do DF, localizadas no Plano Piloto de Brasília. Os resultados mostraram que mesmo com problemas neurológicos, dependendo o tamanho do comprometimento, a aprendizagem da Química é possível, e até agradável, não necessariamente lúdico, mas que permita uma melhor compreensão do que seja a Química, seu objeto de estudo e como ela está presente no cotidiano, assim como a matemática e demais ciências e áreas do conhecimento.
\end{abstract}

Palavras-chave: Discalculia; Estudo de química; Neurociências, Aprendizagem.

\section{Dyscalculia and its Influence on Chemistry Learning: A Case Study in Two High Schools in Distrito Federal - Brazil}

\begin{abstract}
From the emergence of neurosciences, it was discovered how the functioning of the brain influences education, and consequently, learning, and thus help teachers and researchers. In this sense, the present work aims to analyze the relationship between mathematical learning disorders and their consequent influence on the understanding of Chemistry and its learning, since this area of knowledge needs for its understanding of calculus, which is the research hypothesis. . This is a descriptive, quantitative study, carried out with data from 400 high school students from both grades of two public schools in the Federal District, located in the Plano Piloto de Brasília. The results showed that even with neurological problems, depending on the size of the commitment, learning Chemistry is possible, and even pleasant, not necessarily playful, but that allows a better understanding of what Chemistry is, its object of study and how it is present in everyday life, as well as mathematics and other sciences and areas of knowledge.
\end{abstract}

Keywords: Dyscalculia; Learning chemistry; Case study.

\footnotetext{
${ }^{1}$ Professor da Secretaria de Educação do Distrito Federal desde 1985, ex-professor de várias instituições de ensino superior (UPIS, UNEB, CEUB, UDF, UCB etc.), possui graduação em biologia, pedagogia, administração, direito e geografia, especialista em Psicologia Clínica, Análise do Comportamento, Terapia Cognitiva Comportamental e Neurociência e formação em Psicodrama. Mestre em Educação e doutorando em Psicologia. francisco1965@gmail.com.
} 


\title{
Introdução
}

A discalculia é uma das espécies de dificuldades de aprendizagem, só que específica da matemática que pode ser diagnosticada logo cedo por professores ou até mesmo pelos pais. Contudo não deve ser confundida com uma simples dificuldade, ela chega a ser uma patologia que precisa ser tratada. Ela pode ser léxica quando o aluno tem dificuldade na leitura de símbolos matemáticos; verbal, quando o aluno não consegue nomear verbalmente quantidades matemáticas, termos e símbolos, confundindo ou simplesmente não nomeia; a discalculia gráfica: como vivemos em um mundo letrado, muitos têm dificuldade na escrita de símbolos matemáticos ${ }^{2}$, desde simples como os sinais das operações básicas matemáticas até confundir fórmulas complexas, e por último, a discalculia operacional, que é a mais perceptível uma vez que professores e pais podem identificar no individuo se ele consegue ou não executar operações e cálculos numéricos.

Ele é definido topograficamente no DSM V como

\begin{abstract}
Discalculia é um termo alternativo usado em referência a um padrão de dificuldades caracterizado por problemas no processamento de informações numéricas, aprendizagem de fatos aritméticos e realização de cálculos precisos ou fluentes. Se o termo discalculia for usado para especificar esse padrão particular de dificuldades matemáticas, é importante também especificar quaisquer dificuldades adicionais que estejam presentes, tais como dificuldades no raciocínio matemático ou na precisão na leitura de palavras. (DSM-V, 2014, p. 67).
\end{abstract}

Com o surgimento das neurociências descobriu-se como o funcionamento do cérebro influencia a educação, e consequentemente, a aprendizagem e com isso auxiliar professores e pesquisadores. Nesse sentido, o presente trabalho tem como objetivo analisar a relação entre os distúrbios de aprendizagem matemática e sua consequente influência no entendimento da Química e sua aprendizagem uma vez que esta área do conhecimento necessita para o seu entendimento de cálculos, sendo esta a hipótese de pesquisa. Para isso, o modelo teóricometodológico utilizado foi a Teoria das Representações Sociais de Moscovici, e especialmente as contribuições de Doise e seus colaboradores (1993) com sua abordagem societal das Representações Sociais.

Por isso, a pesquisa não pretende aprofundar nos aspectos técnicos dos processos neurológicos que provocam a discalculia, mas sim relatar através de uma pesquisa de campo as dificuldades e as superações de alguns alunos de duas escolas públicas do DF que apresentam

\footnotetext{
2 Uma outra classificação feita por Kosc (1974) apresenta seis tipos de discalculia, acrescentando ainda a practognóstica: dificuldades para enumerar, comparar, manipular objetos reais ou em imagens e practognóstica: dificuldades para enumerar, comparar, manipular objetos reais ou em imagens, por exemplo
}

111 Id on Line Rev. Mult. Psic. V.15, N. 56, p. 110-120, Julho/2021- ISSN 1981-1179

Edição eletrônica em http://idonline.emnuvens.com.br/id 
oficialmente diagnóstico com problemas neurológicos. Para isso, entramos em contatos com diretores das duas escolas no qual tivemos acesso as pastas dos alunos bem como os relatórios médicos que ficam a disposição da escola, tendo cuidado com os aspectos éticos que influenciaram na metodologia.

\section{Aspectos Neurológicos da Discalculia}

Os estudos de neurociência são recentes, mas reporta desde a época de Galeno, que 200 anos do nascimento de Cristo já afirmava que o cérebro tinha áreas especialidades, mas que, durante o final do século XIX (ano 1895) quando Röntegen através do raio X descobriu que havia áreas no cérebro que envolvia aprendizagem de cálculos, o que posteriormente foi estudado e aprofundado pela medicina ( LIMA, ALONSO e PIMENTEL, 2009).

Hoje sabe-se que o lobo pariental é responsável pelo processamento numérico, passível de verificação em exames como o de imagem. Sabe-se também que o número de neurônico afeta no indivíduo ao tentar processar questões numéricas (DEHAENE, 1997,2000). Outro órgão responsável pela resolução de problemas matemáticos é o giro angular, que segundo estudos, consegue recuperar a memória de logo prazo, fazendo que com que haja o reconhecimento de fatos matemáticos. Porém, é preciso assinalar que no transtorno de discalculia alguns processos são afetados, como a velocidade de processamento das informações, a memória de trabalho, as habilidades visuais, a própria linguagem matemática e consequentemente, a química, habilidades psicomotoras e percepções táteis, segundo estudos realizados por Wajnsztejn e Castro (2010).

Contudo, a síndrome da paralisia cerebral e a afasia são as funções neurológicas que mais interferem no processo de aprendizagem, tanto da matemática como da química ou qualquer outra área do conhecimento, mas essa tese é questionada. A primeira porque está relacionada com o mau desenvolvimento ou com a lesão cerebral, quando a criança ainda é imatura, interferindo dessa forma no desenvolvimento psiconeurológico com o comprometimento motor, contudo, não podemos afirmar categoricamente que a paralisia cerebral por si só provoca distúrbios de aprendizagem (MUNIZ, 1972) 


\section{A Discauculina entre jovens de Ensino Médio em duas Escolas Públicas do Distrito Federal - DF}

\section{Participantes}

Este estudo foi realizado com dados de 400 alunos estudantes do ensino médio de ambas as séries de duas escolas públicas do DF, localizadas no Plano Piloto de Brasília. Uma das escolas localizada no "centro", isto é, próximo à estação rodoviária local de Brasília), e outra localizada na asa norte, de idade média de 16 anos ( $\mathrm{DP}=15,7)$ oriundos na sua maioria das regiões administrativas de Brasília ( $\mathrm{N}=388$ ), sendo que 27 fazia estágio após ou antes as aulas ( os anos do segundo e terceiro ano estudavam pela matutino e os do primeiro ano, pelo vespertino). Procuramos uma forma equitativa em relação ao sexo, sendo que $56 \%$ eram do sexo feminino e $46 \%$ do sexo masculino. Todos estudaram o ensino fundamental em escolas públicas e geralmente perto de suas casas.

Em relação às famílias, quase todos de classe baixa (recebiam de um a três salários mínimos), com um número de irmãos igual ou superior a três, em casa própria (72\%), alugada (33\%) e outras. Muitos relataram que seus pais viviam em relação estável, não sendo casados oficialmente, e que tiveram problemas de matemática especialmente durante o oitavo e o novo ano $(\mathrm{N}=320)$.

\section{Procedimentos e medidas}

A coleta de dados ocorreu durante os meses de abril a maio de 2020. As duas escolas foram contatadas diretamente através das equipes gestoras por questão de facilidade uma vez que o pesquisador já foi diretor de uma das escolas e supervisor na outra. Em primeiro lugar foi definido os objetivos da pesquisa e posteriormente foi discutido junto com os diretores e supervisores as formas de coleta de dados. Houve três reuniões antes de estabelecer as estratégias, a primeiro foi para esclarecer os objetivos da pesquisa, as questões éticas e os benefícios e riscos da pesquisa, conforme determinação do Conselho Nacional de Saúde, não houve a necessidade de aprovação por um Conselho de Ética em Pesquisa de Seres Humanos, mas os diretores pediram por escrito um projeto com os objetivos da pesquisa, a metodologia e a assinatura de um termo de responsabilidade com duas cópias. É importante frisar que a ideia de apresentar este documento partiu por parte do pesquisador. 
Após os esclarecimentos e com a finalização da terceira reunião, estabeleceu-se as estratégias de quem seriam os alunos participantes da pesquisa. Como as escolas juntas somamse mais de 4 mil alunos e que a pesquisa foi realizada durante a pandemia, foram estabelecidos alguns protocolos e procedimentos para a coleta dos dados. A primeira foi que nas reuniões pedagógicas (as quartas-feiras) os professores conselheiros entrariam em contato com os representantes de turma través de meios eletrônicos, como Whastsapp e o Google Escola, onde eram realizadas as aulas. No início 1.772 alunos responderam ao questionário que foi enviado pelas redes sociais e realizados pelo google forms.

O Termo de Consentimento e Livre Esclarecimento foi assinado pelos pais dos menores de 18 anos e foi esclarecido que o referido documento seria arquivado em uma pasta por 3 anos. Já os participantes da pesquisa assinaram o Termo e Assentimento (Resolução 466 de 2012 do CNS, capítulo II, número 24)

O questionário aplicado foi divido em três partes: a primeira, com os dados sociodemográficos, explorando idade, cor, sexo, procedência, dados familiares, dados sobre o histórico escolar do aluno (a história de vida escolar). O segundo com três questões abertas, no qual os alunos respondiam sobre quais os problemas que eles enfrentavam na aprendizagem da Química e por último, um instrumento de medição baseada em uma escala estilo liket. Nessa escala eram pedidas algumas contas básicas de matemática como soma com um algarismo, dois e três. Em seguida algumas questões envolvendo raciocínio lógico, com cálculos simples envolvendo balanceamento e equilíbrio químico, bem como cálculo do número atômico, cálculo de molaridade, densidade, número de mols. Este último tinha 20 itens e o tempo de previsão para ser resolvido era de 60 a 80 minutos. Esclarecemos que este instrumento ainda não teve validação, tratando-se simplesmente de um experimento que poderá futuramente ser replicado e adaptado a cada realidade.

Após a aplicação dos instrumentos foram utilizados como critério de exclusão: alunos que não faziam parte da rede pública de ensino do DF e que também não pertenciam a escola (houve alunos que eram de outras instituições, pois os alunos repassaram o questionário para alguns amigos). Outro critério foi o fato de que os alunos para participar da pesquisa tinham que ter laudo médico de discalculia, no qual o Serviço de Orientação Educacional tinha acesso e que o pesquisador conseguiu identificar os 400 alunos que tinham comprovado por neurologistas e/ou psicólogos que tinham discalculia. 
Todas as respostas foram alimentadas no Pacote estatístico SPSS versão 25 por uma empresa júnior de uma universidade pública de Brasília, no qual foram pagos $\mathrm{R} \$ 500,00$ tanto pelo lançamento dos dados como na ajuda da análise.

\section{Resultados}

Com base no modelo de análise quantitativa das Representações Sociais (Doise, Clemente e Orenzi-Cioldi,1993), inicialmente foram identificados os tipos de discalculia e os níveis divididos em séries (primeiro ano, segundo ano, terceiro ano) que os alunos das escolas apresentaram. Em seguida, com base nos dados quantitativos foram analisados as diferenças entre dificuldades de aprendizagem e discalculia e as representações sociais sobre a natureza das dificuldades ou problemas com o objetivo de que com base nos dados, os professores de Química juntamente com os orientadores educacionais e as duas psicólogas escolares pudessem fazer as devidas intervenções para que estes alunos não tivessem prejuízo tanto na sua vida como no rendimento escolar, evitando com isso reprovação. Por fim, com base na metodologia das Representações Sociais, foram analisadas as ancoragens sociais dessas representações

Para responder as perguntas de como os alunos exprimem suas atitudes em relação as dificuldades em Química, seguimos os procedimentos realizados por outros pesquisadores que estudaram sobre discalculia. Alunos que tiveram escores altos $(\mathrm{N}=356)$ foram encaminhados para os profissionais da escola e estes enviados para o a ADOLECENTRO, órgão da secretaria de saúde do DF que trata de alunos com problemas sociais e com problemas escolares

A identificação dos perfis dos alunos foi feito por uma ANOVA no qual no qual foram divididos os alunos por grupos de acordo com (1) sexo - apesar de que essa não era a variável central; (2) por idade e por histórico de dificuldades de aprendizagem em matérias que exigiam cálculos; (3) por series, isto é, entre alunos do primeiro ano, segundo e terceiro. Os resultados demonstraram um efeito multivariado significativo $(4,7888)=321,2$ com $p<0,0001$, indicando este resultado que os perfis dos alunos foram diferentes no que se refere a cognição, problemas neurológicos. Dessa forma, com essa classificação dos estudantes, explica que $98 \%$ da variância total dos posicionamentos na escala utilizada $(\mathrm{V}=8,7)$. Adicionalmente, todas as diferenças foram consideradas homogêneas, tendo em vista os laudos apresentados na escola, então, a análise da média obtidas na amostra mostra que os alunos do primeiro aluno apresentaram maior dificuldade em Química $(98,8 \%)$, segundas pelos alunos do terceiro ano $(88,95)$ e pelos alunos do segundo ano $(85,3 \%)$, confirmando os laudos. Contudo, os próprios laudos afirmaram que 
apesar de alguns alunos apresentarem comprometimento neurológico/cerebral de moderado a alto, eles não apresentavam dificuldades cognitivas significativas. Dos 400 laudos analisados que foram encaminhados para a escola, somente 12 tinham comprometimento grave, mas que não atingia a cognição, necessitando de um ritmo diferenciado de aprendizagem.

\section{Ancoragem sobre a discalculia e os problemas com o entendimento/aprendizagem da Química}

Após o entendimento do que seja Química e Discalculia, analisamos as adesões em relação as crenças sobre a natureza e dificuldades neurológicas, e se há relação entre dificuldade de aprendizagem e/ou discalculia que interferem na aprendizagem da Química, para isso, usamos como critério quanto ao tipo de discalculia ( léxica, verbal, gráfica e operacional) como variáveis preditoras e as crenças em relação as dificuldades de aprendizagem em matemática e consequentemente em Química ( mulheres não são boas em áreas de exatas, exatas são sempre mais difíceis do que humanas, número é algo muito abstrato), para que em seguida fossem feitos dois tipos de regressões. No primeiro, verificou-se apenas as variáveis sociodemográficas, por acha que o ambiente e não fatores biológicos interferem na aprendizagem. No segundo modelo, acrescentamos as crenças sobre os problemas de aprendizagem na área de exatas, no qual o modelo 1 mostrou que crenças aliadas a processos cognitivos embutidos socialmente elevam o carga emocional dos alunos, e com isso, cresce socialmente um medo em relação às ciências exatas, não se questionando modelos metodológicos de aprendizagem ou possibilidade de enfrentar as dificuldades, tentando acabar com a crença de que inteligência é genético. Com isso, percebeu-se que os dados sociodemográficos predizem o tipo de dificuldade que os alunos têm em relação às ciências exatas, principalmente a Química.

As áreas de humanas, apesar de crenças muito forte apresentam como sendo fáceis, porque estão no cotidiano e são facilmente vivenciada, ou caso não sejam vivenciadas, as leituras podem ajudar na compreensão. Esquecem que a matemática é também um tipo de linguagem e que pode ser comparada até mesmo com a língua portuguesa. Contudo, uma intervenção de um profissional específico poderia sanar esse problema, alguém que consiga transmitir que apesar das abstrações, a matemática é constituída de símbolos e que estes símbolos podem ser significados concretos. Contudo, a análise dos coeficientes de regressão indica que alunos que tiveram melhor contato com exatas do que os outros ou que tiveram professores que conseguiam transpor o abstrato para o concreto, tinham menos dificuldades, 
mas outro fator ou variável pode ser importante para esta análise: a capacidade do professor da área de exatas trazer para seus alunos que essas ciências podem ser trazidas também para a realidade, sendo mais concretas ( e.g.CARRAHER, CARRAHER e SCHLIEMAN, 1982. SCHLIEMAN, 2015).

\section{Ancoragem sobre as crenças das dificuldades da aprendizagem da Química}

O senso comum apresenta várias explicações sobre as dificuldades da aprendizagem das ciências sociais, uma delas diz respeito ao fato de que muitos alunos quando criança sofreram algum tipo de trauma e não conseguem aprender. Outra, de caráter mais preconceituoso diz respeito de que indivíduos do sexo feminino têm dificuldade em lidar com número, mas é preciso lembrar, por exemplo dos trabalhos de Marie Curie que em 1903 recebeu o Prêmio Nobel de Física (ela, o marido e Gustave Bémont havia descoberto o Polônio e o Rádio) e alguns anos depois (1911) o Prêmio Nobel de Química, além disso, recentemente, o Prêmio Nobel de Química foi para duas mulheres - Emmanuelle Charpetier e Jennifer Doudna pelo desenvolvimento do Crispr, um método de adição do genoma (PINHEIRO, 2020). Para a Análise desses dados submetemos a uma Análise do Covariência (ANCOVA). O desenho da análise fatorial foi dividido em dois tipos: aprendizagem versus não aprendizagem da Química e crenças negativas versus fatores biológicos versus fatores psicológicos, sendo que o primeiro fator foi inter-sujeitos, enquanto que o segundo foi intra-sujeitos ( uma vez que os alunos encontravam em classes de inclusão), controlados pelas variáveis idade, série, história de vida, grau de comprometimento neurológico. Os resultados mostraram um efeito principal significativo para o comprometimento neurológico, $F(1,334)=11,82$, com $p<0,001, \eta^{2}=0,05$. Em geral os alunos com menor comprometimento neurológico $(\mathrm{M}=4,78 ; \mathrm{DP}=0,78)$ no decorrer do seu histórico dentro das escolas pesquisadas tentem a melhor à medida que outros fatores foram acrescentados como: aumento do suporte psicopedagógico dado pela escola, encaminhamento à unidade de atendimento ao adolescente vinculado a Secretaria de Estado de Saúde, melhor conhecimento dos professores das dificuldades, etc. Notou-se também o efeito negativo das crenças de não aprendizagem $F(6,2376)=6,77, p<0,001, \eta^{2}=0,03$. A análise dos contrates indica que crenças de natureza psicológica a $(M=5,36 ; D P=2,27)$ é mais forte que a na natureza neurológica $(M=4,85 ; D P=2,76)$ da não aprendizagem da Química, $t(649)$ $=7,51, p<0,001$. Não houve diferença significativa entre a crença na natureza biológica $(M=$ $1,14 ; D P=2,49)$ e psicossocial da aprendizagem, $F(2,359)=0,63$, $n s$. 
Finalmente, verificamos em que medida havia diferença de cognição entre os alunos cujos laudos apontavam comprometimento severo de lesão ou de déficit de aprendizagem ou com distúrbios de aprendizagem, o que indicou formas consensuais de representações sociais sobre a natureza, estrutura e manutenção da não aprendizagem de Química, sem haver contudo relação com discalculia, mas sim com metodologias aplicadas pelos professores, mas devido aos serviços oferecidos pelas escolas ( e.g. SOE, Atendimento Especializados a Alunos) estes problemas foram resolvidos, havendo um progresso no ingresso dos alunos desde o primeiro ano até o terceiro ano do ensino médio.

\section{Discussão}

Os resultados desse estudo respondem a tese central: a matemática é fundamental na aprendizagem da Química porque esta para ser entendida e melhor compreendida necessidade de cálculos, desde o começo introdutório, com cálculos simples de peso atômico, massa atômica densidade ( que exige uma simples divisão entre massa e volume), pressão ( também o aluno tem que saber dividir força por área) que tem início logo no 9 ano do ensino fundamental, até chegar a cálculos mais complicados como balanceamento químico, cálculo de mol, cálculo estequiométrico, cálculo das constantes de equilíbrio, reações químicas etc.

Em síntese, apesar dos estudos neurológicos e os avanços das neurociências, sabe-se que tanto a discalculia como dificuldades de aprendizagem de Química ou qualquer que seja a área podem ser superadas, não há um determinismo.

Embora a investigação tenha mostrado que há um grande número de alunos com transtornos de aprendizagem na área de exatas, também existe alunos com transtornos nas áreas de humanas e que o cérebro desempenha papel importante para entender esses mecanismos. Também não podemos esquecer as contribuições históricas trazidas por Röenten no final do século XIX e que trouxe uma luz para o progresso que revolucionou a medicina.

\section{Conclusões}

A maioria das pesquisas sobre discalculia recaem sobre aspectos puramente pedagógico, apontando de forma simples que se tratada de um problema de aprendizagem, ou de cunho psicológico ou neuropsicológico, um pouco mais apurado e que traz elementos mais científicos para o seu entendimento. Mas, no que diz respeito ao modelo médico este recai aos problemas 
de aprendizagem da Química com base puramente biológica, não levando em conta elementos sociais e até mesmo a história de vida do aluno. Há sim alunos que comprovadamente têm comprometimento neurológico, mas estes têm atendimento escolar especializado e estão colocados em sala de aulas juntamente com outros alunos - a educação inclusiva - com o objetivo de que, tanto o aluno com problemas neurológico possam socializar suas dificuldades e os seus conhecimentos sobre o seu problema, possa compartilhar com os demais alunos, que apesar de não terem problemas neurológicos, têm sim problemas de aprendizagem em Química, mostrando que o encontro entre esses dois tipos de alunos podem ser ricos e a aprendizagem possa ser mais eficiente. Nas duas escolas pesquisadas seguem plenamente o que a legislação determina, e apesar de serem escolas públicas, todos os alunos recebem atendimento especial, sem distinção e discriminação.

A pesquisa mostrou que mesmo com problemas neurológicos, dependendo o tamanho do comprometimento, a aprendizagem da Química é possível, e até agradável, não necessariamente lúdico, mas que permita uma melhor compreensão do que seja a Química, seu objeto de estudo e como ela está presente no cotidiano, assim como a matemática e demais ciências e áreas do conhecimento.

Da mesma forma, a pesquisa mostrou que há limitações na pesquisa e a necessidade de estudos empíricos que possam estudar mais profundamente as questões que envolvem as dificuldades na aprendizagem de Química que não envolvam apenas os aspectos neurobiológicos e além do mais, a pesquisa mostrou que há poucos estudos a respeito desse objeto de estudo, e que há muitos estudos envolvendo dificuldade de aprendizagem ou até mesmo sobre discalculia, necessitando estudos mais profundos a respeito da relação entre neurociência, educação e aprendizagem de Química.

Frisamos e enfatizamos que o diálogo entre neurociências e aprendizagem no campo da educação é importante para a prática docente. Os conceitos e os conhecimentos das neurociências podem servir de norte para transtornos de aprendizagem, no nosso caso específico, o ensino da Química, mesmo que haja sérios comprometimentos é possível a aprendizagem como pesquisas recentes demonstram isso. Além disso, indicamos como fonte de tratamento além das psicoterapias medicamentosas, a terapia analítico comportamental que pode proporcionar uma aprendizagem da química de maneira fácil, criativa e dinâmica. Uma verdadeira aprendizagem da Química só se realiza (1) por meio da contextualização; (2) o entendimento das transformações químicas; (3) através de estímulos e um desenvolvimento cognitivo; (3) formação para a cidadania; (4) maior conhecimento dos professores do 
funcionamento do cérebro, seja na graduação seja em cursos de especialização ou até mesmo em mestrados ou doutorados), etc.

\section{Referências}

CARRAHER, Terezinha Nunes, CARRAHER, David William e SCHLIEMAN, Ana Lúcia. Na vida dez;na escola zero: os contextos culturais da aprendizagem matemática. Cadernos de Pesquisa, V. 42, pp. 79-96, 1982. Disponível em https://professores.im-uff.mat.br.

DEHAENE, S. The number sense. Oxford, UK: Oxford University Press, 1997.

DEHAENE, S. Cerebral bases of number processing and calculation. In: GAZZANIGA, M.S.The new cognitive neuroscience. 2 ed. Institute of Technology, Massachussets, 2000.

DOISE, Willem., CLÉMENCE, Alain., e LORENZI-CIOLI, Fábio.The quantitative analysis of social representations. Hempel Hempstead: Harvester Wheatsheaf, 1993.

DSM-V. Manual Diagnóstico e Estatístico de Transtornos Mentais: DSM-V/ (American Psychiatric Association). 5aed. Porto Alegre: Artmed, 2014.

LIMA, Rodrigo da Silva, AFONSO, Júlio Carlos e PIMENTEL, L. C. Ferreira. Raios X: fascinação, medo e ciência. Química nova, Vol. 32 n. 1, pp. 263-270, 2009.

KOSC, Ladislava. Developmental Dyscalculia. Journal of Learning Disabilities. Vol.7, Número 3, 1974.

MOSCOVICI, Serge. La psychanalyse, son image, son public, University Presses of France, 1976.

MUNIZ, Paulo C. Aspectos neuropsiquiátricos do atendimento escolar na paralisia cerebral. Revista Brasileira de Estudos Pedagógicos. 58(127): 87-91, Jul-Set. 1972.

PINEHEIRO, Lara. Nobel de Química 2020 vai para Emmanuelle Charpentier e Jennifer Douddna. Portal G1, de 07.10.2020, Rio de Janeiro, Ciência e Saúde. Disponível em https://g1.globo.com/cienciae-saude/noticia/2020/10/07.

SCHLIEMAN, Ana Lúcia. Na vida dez, na escola zero. 16ª.ed. São Paulo: Cortez, 2015.

WAJNSZTEJN, Rubens.; CASTRO, V. T. Discalculia ou transtorno específico das habilidades matemáticas. In: VALLE, L. E. R.; ASSUMPÇÃO, F.; WAJNSZTEJN, R.; DINIZ, L. F. M. (Orgs.). Aprendizagem na atualidade: neuropsicologia e desenvolvimento na inclusão. São Paulo: Novo conceito Editora, 2010

\section{Como citar este Artigo (ABNT):}

SOUSA, José Francisco de. A Discalculia e sua Influência na Aprendizagem da Química: Um Estudo de Caso em duas Escolas de Ensino Médio no Distrito Federal - DF. Id on Line Rev.Mult. Psic., Julho/2021, vol.15, n.56, p. 110-120, ISSN: 1981-1179.

Recebido: 04/06/2021; Aceito: 09/06/2021. 